\title{
Integración de las terapias en el currículo enfermero
}

Julia López Ruiz

Profesora de Enfermería. Departamento de Enfermería Fundamental y Médico-Quirúrgica. Escuela Universitaria de Enfermería.

Universidad de Barcelona. Barcelona. España.

El interés en potenciar las terapias complementarias (TC ${ }^{\mathrm{a}}$ y que éstas formen parte de los conocimientos y la práctica enfermera es cada vez más creciente, lo que me ha llevado a indagar qué se está escribiendo al respecto y a cuestionarme sobre las orientaciones que se siguen para que las TC estén presentes en la formación básica enfermera, paso previo para que formen parte de los cuidados enfermeros.

LA INTEGRACIÓN de las prácticas holísticas en la formación enfermera permitiría tener "una nueva generación de enfermeras holísticas cuidadoras", como dice Sandor ${ }^{1}$. Esto provocaría cambios positivos en la manera de ofrecer los cuidados y en la manera de relacionarnos con las personas cuidadas. Desde un planteamiento muy positivo, quiero confiar en que así sea.

Desde que en el 2000-2001 el Grupo de Terapias del Colegio de Enfermería de Barcelona ${ }^{2}$ se planteó elaborar el llamado "Documento Marco", hasta el momento presente, se han producido cambios relevantes en aspectos relacionados con la regulación de las TC y su práctica; las perspectivas ahora son algo más alentadoras, a pesar de las numerosas dificultades que aún han de superarse.

En nuestra sociedad occidental, en los profesionales de la salud y en sus organizaciones se refleja la preocupación por que las TC sean ofertadas a los usuarios con las mayores garantías de calidad y seguridad, lo que plantea determinadas exigencias en el ámbito de la formación y de la investigación, aspecto que debe tener un reflejo en las políticas sanitarias, tal como lo plantea la $\mathrm{OMS}^{3}$. Integrar las TC en la praxis y en la formación es una de las cuestiones emergentes en el mundo de la salud y requiere respuestas de los formadores de sus profesionales. Plantear cómo pueden integrarse en la formación básica enfermera es uno de los objetivos de este escrito. Espero que mi aportación contribuya en algo a que las TC se incluyan en el currículo enfermero. Sé que en un porcentaje muy elevado de las escuelas de enfermería de España ya se incluyen algunos conocimientos, pero se hace por el compromiso de algunos profesores, no de todo el profesorado, y esta inclusión se realiza con carácter de asignatura optativa y no siempre se llega a todos los alumnos.

El desarrollo de esta exposición intenta dar respuesta a las siguientes cuestiones:

- Qué justifica la inclusión de las TC en el currículo enfermero.

- Qué planteamientos se hacen en el ámbito enfermero.

- Posibilidades de integrar las TC en el currículo enfermero.

${ }^{a}$ En el artículo preferentemente se utiliza el término TC (terapias complementarias). Considero que cuando nos referimos a las TC, ya está implícita la idea de que son terapias naturales. El término "instrumentos complementarios de los cuidados enfermeros" (ICCE) requiere haber asumido un determinado enfoque, por lo que se utiliza más restringidamente. El término "medicinas complementarias y alternativas" (MCA) no se utiliza en el ámbito de la enfermería, pero sí en campos en los que el término es más apropiado, como en los planteamientos médicos.

- Condiciones exigibles/recomendaciones.

Se incluye también la bibliografía consultada, que permitirá ampliar información sobre las ideas que aquí se ofrecen.

\section{QUÉ JUSTIFICA LA INCLUSIÓN DE LAS TERAPIAS COMPLEMENTARIAS EN EL CURRÍCULO ENFERMERO}

Como dicen algunas autoras, se ha de justificar y razonar por qué deben introducirse estos conocimientos dentro de la formación básica enfermera ${ }^{4}$. El planteamiento debería hacerse considerando primero los intereses, las necesidades, los resultados o los beneficios para el usuario y no tanto desde el punto de vista del interés de los profesionales 5,6 .

Entre los argumentos que pueden considerarse, fijamos la atención en aspectos relacionados con la población, con la salud y con las tendencias actuales respecto a las TC y respecto a la formación.

\section{Responder a las demandas de la población}

- Las tasas de población que en los diferentes países recurren a las TC es una de las razones más repetidas; tasas que siguen en aumento ${ }^{1,3,7-9}$.

- Tenemos una sociedad dinámica, abierta, cambiante, multicultural, más globalizada e informada, que toma conciencia del valor de lo natural y de la importancia de la ecología.

- Los consumidores y proveedores de la salud hacen opciones por enfoques "holísticos y/o integrales" que ofrecen concepciones respecto a la persona, la salud, el entorno y la atención de salud que se presta, muy diferentes a las convencionales. 


\section{complementarias}

edición española

- La globalidad de la persona -como más y diferente a la suma de sus partes-, su unicidad, su interacción continua y permanente con el entorno, etc.

- La salud como proceso, experiencia, un medio que implica a toda la vida de la persona. Con frecuencia se considera la atención de la salud muy

despersonalizada, parcializada, etc.

- Muchas personas están tomando conciencia de la responsabilidad sobre su salud, sus derechos, su capacidad para decidir; de los servicios de salud que se les ofrecen, etc. Estas prácticas favorecen esa toma de conciencia.

\section{Razones relacionadas con la salud}

- Los usuarios encuentran, en estos medios, determinados beneficios, como un enfoque de atención más centrado en sus intereses, experimentan relajación, bienestar, autocontrol en las situaciones de ansiedad, etc.

- Se destaca que el uso de determinadas TC constituye un potencial para el fomento y la promoción de la salud, porque están ayudando a generar principios y estilos de vida más sanos 8,10 - Son un recurso natural para potenciar el autocuidado (muy importante para los profesionales de la salud) y para el aumento de las capacidades defensivas. - Evidencia de que la salud engloba e involucra a todos los aspectos integrantes de la vida de las personas -mente, cuerpo, espíritu, entorno y calidad de la atención-; los cuidados de salud deben apoyar la globalidad de la persona ${ }^{9}$.

- Aumenta la convicción de muchas profesionales de que la medicina alopática, hoy, no puede dar respuestas a todos los problemas de salud de la población, tales como situaciones de cronicidad, control del dolor, envejecimiento y soledad, etc. ${ }^{9,11}$

- El elevado coste de la tecnomedicina, de la farmacología y sus efectos secundarios.

- Se analiza que la oferta de estos medios debería empezar a considerarse desde el punto de vista económico, sobre todo para afrontar muchos problemas crónicos de salud ${ }^{11,12}$. Destacan tanto la miopía como la visión progresista y de futuro de determinados gestores en relación con la utilización de estos medios en los sistemas de salud.

\section{Tendencias actuales respecto a las TC}

- En la última década, muchas instituciones han promovido declaraciones, recomendaciones y políticas relacionadas con la regulación y acreditación en TC/MCA. Implica a gobiernos, organizaciones, colegios profesionales, centros, asociaciones y publicaciones ${ }^{b}$ - La integración de las TC dentro de los sistemas asistenciales de salud está en el horizonte futuro. Los esfuerzos por compaginar aportaciones de la MCA con los procedimientos y práctica de la medicina alopática están dando lugar a la llamada "medicina integrativa".

En este enfoque "integrativo" se promueve que se tengan en consideración una buena información de los intereses del cliente y su derecho de elección. Empiezan a producirse experiencias que incidirán en diferentes grupos de profesionales de la salud y, por supuesto, en los profesionales enfermeros.

Diferentes autores hacen aportaciones al respecto:

- David Katz dice 13: "Dado el interés de los usuarios por las MCA, un sistema sanitario que no tenga en cuenta estas modalidades no puede estar realmente centrado en la persona".

"Imaginar las ventajas de una atención integrativa puede ser el primer paso para tomar conciencia de que la MCA también incluye terapias seguras y eficaces; hay que actuar con cautela, pero se ha de ir por el camino de la integración; es prioritario que ambas modalidades terapéuticas convivan en armonía...".

- Según J. Gordon ${ }^{9}$, ante las limitaciones de la medicina convencional se está buscado un enfoque integral que tenga en cuenta todos los modos de curación y la globalidad de la persona; enfoque que está ganando cada vez más partidarios entre los profesionales de la salud. Se confía en que la integración se haga con el apoyo o de la mano del conocimiento adquirido por profesionales científicamente entrenados en la investigación de la medicina alopática.

- Sandor ${ }^{1}$ comenta que, en algunos centros de Estados Unidos, gestores futuristas contratan a profesionales enfermeros con conocimientos y acreditación en TC, y que éstos están siendo mejor retribuidos. Y diferentes

bPor citar algunos: documentos de la OMS; recomendaciones del Parlamento Europeo; informe de la Casa de los Lores (Reino Unido); informe de la Comisión de la Casa Blanca; The NCCAM-Centro Nacional para la Medicina Complementaria y Alternativa (Estados Unidos); decreto de la Generalitat de Cataluña sobre la regulación de las terapias naturales; documentos de la OIIQ; documento del Royal College of Nursing; estándares de la práctica de la Asociación de Enfermeras Holísticas Americana (AHNA); documento Marco del Grup de Teràpies Complementàries del Col-legi Oficial d'Infermeria de Barcelona; etc. 
compañías de seguros sanitarios incluyen en sus servicios determinadas TC.

- Rankin-Box ${ }^{12}$ opina que en la próxima década se espera una sinergia simbiótica entre ambas modalidades terapéuticas, reconocimiento e integración, y mayor acercamiento cultural, considerando el concepto de aldea global en el que ahora vivimos y la sociedad multicultural.

\section{Orientaciones o tendencias respecto a la formación}

Integrar las TC en los servicios del sistema de salud exige hacerlo con criterios de racionalidad, seguridad y eficacia, lo que implica comprometerse en la formación de los profesionales de la salud y dedicar recursos a la investigación.

El llamamiento a que los profesionales de la salud reciban formación en medicinas complementarias y alternativas (MCA) y TC se produce desde hace más de una década:

- En 1994 el Dr. P. Fisher ${ }^{14}$, en uno de los artículos más citados y publicado en el British Medical Journal, ya recomendaba la armonización de la enseñanza y su regulación entre los diferentes Estados miembros de la Comunidad Europea (CE).

- En 1994 (Informe Lanoye) la CE ya pedía que se estudiase la situación en cada uno de sus Estados, para limitar las diferencias y para que los profesionales pudieran circular libremente. Según uno de sus miembros (Ahern ${ }^{13}$ ) aún se sigue pidiendo la armonización, que los profesionales que practican las TC estén bien formados y acreditados y que se investigue en este campo.

- En 1995 la OMS ${ }^{15}$ ya pedía que las enfermeras tuviesen conocimientos en lo que ella llama "medicina tradicional" (MT) para asesorar a los usuarios, y más concretamente, en el documento "Estrategia de la OMS sobre Medicina Tradicional 2002-2005"3 respecto a la formación se dice que:

- Es necesario asegurarse de que los conocimientos, la calificación y la formación de los proveedores de las MCA son adecuados. La formación ha de asegurar, a proveedores de la MCA y de la alopática, que ambas se complementan en la atención de la salud.
- Debe asegurarse que los grados de formación de profesionales de la salud incluyan componentes de MCA.

- Han de implementarse sistemas para que las personas cualificadas puedan ofrecerlas.

Y para ello pide:

- Compromiso político y sostenible, a los gobiernos.

- El apoyo y la creación de programas de formación para los profesionales de la salud, a las instituciones académicas y de enseñanza.

- El apoyo de las asociaciones profesionales.

- Estudios en Estados Unidos, según J. Gordon $^{\mathrm{c},} 9$, indican que aproximadamente el $64 \%$ de las facultades de medicina ofrecen formación electiva en MCA, y muchos médicos en ejercicio piden también entrenarse en este campo.

- Y, según Helms ${ }^{10}$, la Universidad de California en San Francisco tiene un centro para la "medicina integrativa".

- Estudios realizados en escuelas de enfermería reflejan que se están introduciendo estos conocimientos en sus programas. Un artículo de 2003 indica que, según las variables que se consideren, el porcentaje está entre el 64 y el 84\% sobre un total de 125 escuelas que responden al estudio ${ }^{16}$. La integración de las TC en los planes de estudio es la tendencia nacional en Estados Unidos, donde además se ofrecen programas de formación continuada a los profesionales de la práctica ${ }^{1}$.

- La opinión de los estudiantes de enfermería y también de otras disciplinas, fuera y dentro de nuestro medio, es que estos conocimientos se incluyan en el currículo básico; según algunos, hasta el $82 \%$ de estudiantes lo considera necesario ${ }^{8,17}$.

- La opinión de los alumnos de enfermería que en "asignaturas optativas" reciben conocimientos de $\mathrm{TC}$ en la escuela de enfermería de la Universidad de Barcelona, es que se ofrezcan a todos los alumnos y que deberían estar incluidos como asignaturas obligatorias. (Es lo que hemos recogido también en mi experiencia, impartiendo esta asignatura desde 1993.)

Si se consideran todos los factores expuestos y se valora el horizonte de la integración de las TC en los servicios de salud, se ve que la universidad, las facultades y las escuelas tienen una importante responsabilidad para que estos conocimientos se incluyan en la formación de los futuros profesionales de la salud, y, como ya hemos dicho, es exigible un compromiso para que los usuarios reciban estos servicios con suficientes garantías de calidad.

\section{QUÉ PLANTEAMIENTOS SE HACEN EN EL ÁMBITO ENFERMERO}

La historia de la enfermería demuestra la preocupación de sus profesionales por dar respuesta a los cambios y a las necesidades de la sociedad; cómo han sabido desarrollar conocimientos y estrategias para responder en todos los tiempos. Como muchas veces se ha dicho, los profesionales de enfermería tenemos un papel relevante en el cuidado de la salud y también en el mantenimiento del sistema sanitario, y porque estamos cerca de la persona cuidada, tenemos la oportunidad de orientar, informar y educar a los clientes y de ofrecerles las TC en nuestros servicios de cuidados; por eso se demanda formación y regularización.

\section{Una mirada a nuestros orígenes. Reevaluar enseñanzas}

Como todos sabemos, los cuidados enfermeros en sus orígenes y en el inicio de su profesionalización han tenido un claro enfoque naturista: evoquemos cómo se ofrecieron los primeros cuidados, con qué y para qué, tal como refleja Colliere ${ }^{18}$. Volvamos la mirada hacia F. Nightingale (1858), de quien se dice fue mística, visionaria, reformista, sanadora, medioambientalista, científica, etc. Según sus enseñanzas, "sólo la naturaleza cura; lo que tiene que hacer la enfermera es poner a la persona en las mejores condiciones para que la naturaleza actúe".

Comparto la opinión de algunas autoras enfermeras de que: 
- "Hemos de recuperar el espíritu de F. Nightingale, las orientaciones, que nunca debiéramos haber olvidado o reemplazado totalmente por un enfoque excesivamente tecnologizado y medicalizado"10,19. Es el momento de reconsiderar sus enseñanzas, y de reevaluar cómo deberían educarse las enfermeras. Y aunque la fisonomía de los modernos cuidados de salud ha cambiado, la esencia del mensaje de F. Nightingale sigue siendo la misma y nos conduce hacia los cuidados holísticos.

\section{El enfoque filosófico y holístico}

El planteamiento filosófico enfermero ha llevado a la enfermería a un continuo cuestionarse sus creencias, sus valores, sus conocimientos, su centro de interés, su razón de ser, su práctica, etc., y el enfoque particular que la singulariza y le permite diferenciarse del resto de disciplinas.

Ponemos de relieve que el enfoque enfermero se apoya en la "teoría holística", aunque no siempre es asumido o comprendido, porque, de asumirlo, tendríamos respuestas algo diferentes respecto a cuestiones básicas y esenciales de la teoría de enfermería: cómo se concibe la persona, la salud, el entorno y el cuidado, etc. Asumir una teoría es mucho más que un ejercicio educativo e intelectual. La teoría debe impactar en la vida profesional y también en lo personal; fortalece y guía el propósito, el significado y la misión encomendada.

La práctica de la enfermería holística se fundamenta en el conocimiento enfermero desde la perspectiva de la globalidad, del más y diferente a la suma de partes, en la experiencia, la intuición y la creatividad, la investigación. La enfermería holística es un instrumento facilitador dentro del proceso de salud, considera de manera particular la experiencia subjetiva del cliente en torno a su salud, sus creencias y sus valores, su espiritualidad $^{20}$. Desde este enfoque, los profesionales de enfermería se comprometen con el cuidado que apoya y transforma.

Como se ha planteado en numerosas ocasiones, aspectos del enfoque en que se sitúan las TC tienen mucho que ver con ese enfoque holístico. Así se recoge en diferentes teorías de enfermería, como las de M. Rogers, J. Watson, M. Newman, etc. ${ }^{7,21}$. De manera particular, la Asociación Holística de Enfermería está incidiendo en que las TC formen parte de los cuidados enfermeros. Así también lo recogen otros grupos, remarcando que las TC son instrumentos de cuidados 22,23 .

\section{Clasificación de las intervenciones enfermeras}

Como sabemos, las clasificaciones internacionales de enfermería -la de diagnósticos enfermeros (DE), la de las intervenciones enfermeras (NIC) y la de los resultados (NOC) - se han desarrollado con la finalidad de unificar criterios en el lenguaje, determinar cuáles son los problemas que resuelven las enfermeras y las intervenciones que deben reconocérseles como propias, autónomas e independientes.

En la NIC, que configura la práctica del dominio enfermero, se incluyen ya diferentes TC, y se pone de relieve que estas modalidades terapéuticas ya han sido incluidas dentro de su competencia. La importancia que se concede a la NIC refuerza la justificación de que las TC forman parte de la esencia de enfermería, pertenecen a las acciones derivadas del juicio clínico profesional y se integran dentro del llamado "proceso enfermero" 7,24 . Según Snyder ${ }^{25}$, en el sistema de salud actual, en el que todo debe ser documentado, las enfermeras, al incluir las TC en las clasificaciones enfermeras, han sabido resolver ese desafío de documentarlas y normalizarlas en su práctica.

En la NIC ${ }^{26}$ se incluyen: visualización, respiración guiada, relajación, masaje, toque terapéutico, musicoterapia, etc. Algunas autoras valoran positivamente que las enfermeras destaquen estas modalidades de terapias no invasivas como intervenciones de enfermería, porque permiten que se pueda disponer de una gran caja de "herramientas o instrumentos de los cuidados" que pueden facilitar la inclusión de las TC en la formación básica y en la formación continuada y el llevarlas a la práctica enfermera.

\section{POSIBILIDADES DE INTEGRAR LAS TERAPIAS COMPLEMENTARIAS EN EL CURRÍCULO ENFERMERO}

Las ideas presentadas hasta aquí, entre otras, son las que han llevado a que en centros de formación de enfermería se estén revisando currículos para facilitar a los alumnos experiencias formales de educación, necesarias para integrar las $\mathrm{TC}^{1,16,27}$. Pero integrar estos conocimientos en planes de estudio que ya están saturados, como dice una autora, resulta un auténtico desafío 8,10 Exige reflexionar sobre qué enseñar, cuándo, qué meta se desea conseguir y cómo enseñar las TC.

\section{Qué integrar: cuna simple técnica o un acercamiento fillosófico?}

La consideración sobre las posibilidades que se nos ofrecen, para que todos los alumnos de enfermería tengan formación en TC, me ha llevado a plantear lo que puede considerarse como un punto de partida que avanzaría hacia un enfoque totalmente holístico (y voy a permitirme ser un poco utópica). Exige cuestionarse sobre la meta a conseguir y que no es ajena al enfoque que se tiene de la disciplina enfermera. El reto hace que nos planteemos si se desea avanzar por integrar estos conocimientos desde un enfoque filosófico y holístico, o lo que integremos son unas simples técnicas ${ }^{24}$.

El planteamiento va desde que:

- La formación básica, que por lo general tiene muchos planteamientos de la medicina convencional y algunas pinceladas de planteamientos holísticos, incluya el conocimiento de alguna TC. Este planteamiento es factible incluyendo alguna de las TC de la NIC. Permitiría al alumno disponer de la "caja de herramientas" que pueden utilizarse con un criterio profesional.

Hasta que:

- La formación básica tenga un enfoque teórico y práctico fundamentado y dirigido por la teoría holística y naturista, que permita al alumno asumir sus principios filosóficos, sus valores, y desarrollar la práctica de cuidados siguiendo los planteamientos de esta teoría, incorporando alguno de los modelos conceptuales enfermeros que ya incluyen la conceptualización y la práctica de cuidados holísticos y que ya integran las TC como cuidados enfermeros.

El primer planteamiento tiene que ver con un "modelo ecléctico" como el que se sigue en muchos centros, que 
combina el enfoque medicalizado -que durante tiempo ha tenido la enfermeríacon algunos planteamientos teóricos enfermeros. Puede permitir avanzar hacia un enfoque integrativo que incorpora modalidades terapéuticas diversas, como si se incorporasen "pedacitos" del enfoque holístico al sistema convencional que hoy se tiene ${ }^{24}$. Esto puede considerarse, como ya he dicho, un punto de partida para ir avanzando hacia un planteamiento totalmente holístico, como el que siguen las enfermeras holísticas, y el fundamento de su asociación y de su práctica tiene el compromiso de guiarse por los estándares holísticos de enfermería ${ }^{28}$.

Ambos planteamientos exigen un compromiso tanto del centro como del profesorado, aunque situarse en cualquiera de ellos dependerá de la orientación que se decida dar a la formación enfermera y de la capacidad de cambio de los centros. Dichos planteamientos cuestionan los enfoques paradigmáticos desde los que se plantea la enseñanza y la práctica. Indican si nos movemos por paradigmas del determinismo y el objetivismo -más centrados en la enfermedad y sus tratamientos, etc.- o nos movemos por el paradigma de la complejidad, la subjetividad, la creatividad, etc. -que incluye los contextos sociales, culturales y las vivencias de las personasNaturalmente, esto implica qué enseñar y cómo enseñar.

\section{Qué posibilidades hay, según el nuevo plan de estudios}

Cualquier planteamiento debería ser coherente con el plan de estudios. Como se sabe, en España se está en proceso de cambio de los estudios de enfermería, para unificar criterios con la CE; se pasa del título de diplomado al título de grado en enfermería, con posibilidades de continuar estudios de máster y de doctorado. ¡Gran avance!

Revisando documentación al respecto, he llegado a la conclusión de que en lo referente al enfoque filosófico y teórico se mantienen planteamientos que no contemplan estos conocimientos, y ni siquiera citan las clasificaciones internacionales enfermeras.

La inclusión de estos conocimientos dependerá mucho de cómo el centro y los profesores lo planteen, si hay compromiso por facilitar las experiencias en TC, y si se buscan estrategias para avanzar. Podrían facilitarse experiencias en asignaturas propias de la universidad, de manera que se llegue a todos los alumnos, y no quede sólo en asignaturas optativas -como ocurre ahora-, sistema que difícilmente permite llegar a todos los alumnos. Se podría profundizar en el enfoque filosófico y holístico/naturista en los "fundamentos teóricos" de la disciplina; incluir TC en la planificación de cuidados cuando realiza las experiencias prácticas, en la aplicación del proceso enfermero. Incluso puede promoverse que los alumnos realicen su trabajo de fin de grado en esta materia.

En la formación básica se fundamentan los conocimientos y se facilitan las primeras experiencias; es la posibilidad de abrir camino para que después el profesional pueda seguir una formación más amplia, especializada, en cursos de posgrado, seleccionando aquellas TC en las que se esté más interesado, dependiendo de los ámbitos en que desarrolle la práctica enfermera. El compromiso del centro y de los profesores debería llegar también a ofrecer posibilidades de formación en TC a los posgraduados, alcanzar diferentes niveles de entrenamiento en cursos de posgrado o máster, y si fuese necesario facilitar la "acreditación" en colaboración con las organizaciones profesionales.

\section{CONDICIONES EXIGIBLES PARA INTRODUCIR LAS TERAPIAS COMPLEMENTARIAS EN EL CURRÍCULO BÁSICO}

Responder a las demandas de la población, respetar las preferencias de los clientes, sus necesidades, debería ser un compromiso. A propósito, me permito recordar una idea de Virginia Henderson: "Si las enfermeras no hacen lo que deben hacer, personas menos preparadas lo harán”. ¡Se ha de evitar que esto pase!

La experiencia y la formación que han desarrollado muchos profesionales de la salud, en este ámbito, debería aprovecharse. Por eso voy a recoger algunas de las recomendaciones al respecto y que pueden considerarse condiciones mínimas exigibles.

\section{Implicación del centro} y de los profesores

- Que sea un planteamiento del centro que implique a todos y no sea cuestión de unos pocos profesores en particular. - Que los profesores estén preparados y comprometidos.

- Según Helms ${ }^{10}$, una de las dificultades más importantes para la integración de las TC en la formación básica es la falta de conocimiento y de experiencia de los profesores.

- Por eso, como primera estrategia se proponen cursos para formar a los profesores $^{16}$.

- Que en el centro se disponga de medios, de documentación y de material suficiente.

\section{Que se diseñen las metas a conseguir}

- Qué enfoque se puede tener, considerando las condiciones del centro y de formación del profesorado; qué sería recomendable para la formación básica enfermera y para la formación posbásica y continuada.

- Seleccionar:

- Las TC más afines a la disciplina, con la propia misión de cuidados, y que sean aplicables en la práctica diaria.

- Las TC más documentadas y que ya están aceptadas por los profesionales. Pueden incluirse las que ya forman parte de las "intervenciones autónomas" en la NIC.

\section{Qué debería facilitarse al alumno en la formación básica} - La oportunidad de reflexionar sobre la filosofía y enfoque -el holismo- en el que se enmarcan las TC. Cómo integrarlas en nuestra propia disciplina y cómo permitir que mejoren el servicio y desarrollen su función social.

- Proporcionar tiempo y espacio para que el alumno tenga experiencias concretas en prestar y recibir cuidados mediante alguna de las TC.

- La adquisición de conocimiento teórico y práctico. Facilitar protocolos y guías de actuación, y siempre desde la propia metodología de la disciplina.

- Descubrir cómo las TC son un medio para el propio autocuidado.

- Potenciar que el alumno sea crítico con estos medios; que se cuestione, se 
documente, que considere sus capacidades y habilidades; éstas se aprenden, no suelen ser innatas.

- Apoyar el trabajo individual, la autorreflexión escrita, dialogar sobre la experiencia. Si fuera posible, facilitar que el alumno escoja la TC con la que quiere trabajar.

- Proporcionar al alumno pautas claras y coherentes, reflexionando sobre cómo y cuándo debe utilizar una TC. Por ejemplo:

- Respeto al derecho del usuario a realizar opciones y a ser informado. Que primero escuchen los motivos y expectativas de las personas que acuden a una TC.

- Que observen cómo las personas siempre nos manifestamos y actuamos holísticamente -aunque como profesionales no asumamos ese enfoque.

- Que tomen conciencia de que para integrar las TC en su práctica siempre deben guiarse por las exigencias del "código ético profesional".

- Que se guíen siempre por las necesidades del cliente; deben contar con el equipo interdisciplinar.

- Que puedan dialogar sobre las recomendaciones que se nos ofrecen a la hora de poner en práctica una TC, y facilitarles la documentación de enfermería al respecto (p. ej., la que nos ofrece la OIIQ; la guía del RCN; los criterios que ofrece Rankin-Box ${ }^{29}$; o los criterios de los estándares holísticos de enfermería, etc.; todos ofrecen indicaciones que les ayudarán a tener criterios claros de actuación al respecto).

\section{CONCLUSIÓN}

Quiero terminar manifestando que soy una enfermera convencida de que asumir este enfoque holístico en el que se enmarcan las TC enriquece nuestra función de cuidar, y que, a pesar de las dificultades, espero que la integración de las TC en la formación y en la práctica enfermera sea una realidad.

Muchas enfermeras han puesto de relieve el arte de la enfermería como la capacidad que, a través de los tiempos, ha tenido para dar respuesta a las necesidades de la población y asumir nuevos retos. Como dice Sandor ${ }^{1}$, se abren nuevos caminos a los formadores: preparar el futuro hacia una "NUEVA GENERACIÓN DE ENFERMERAS HOLÍSTICAS".

\section{Bibliografía}

1. Sandor MK. Educating the next generation of healers. Journal of Holistic Nursing. 2005;23:117-9

2. Grupo de Terapias Complementarias del Colegio Oficial de Enfermería de Barcelona. Instrumentos Complementarios de los Cuidados de Enfermería. Documento Marco. Barcelona: COIB; 2004

3. OMS. Estrategias de la OMS sobre Medicina Tradicional 2002-2005. Ginebra: OMS; 2002.

4. Reed FC, Pettigrew AC, King MO. Alternative and complementary therapies in nursing curricula. J Nurs Educ. 2000;39:133-9.

5. Johnson G. Should nurses practise complementary therapies? Complementary Therapies Nursing \& Midwifery. 2000;6:120-3.

6. Royal College of Nursing. Complementary therapies in nursing, midwifery and health visiting practice. RCN guidance on integrating complementary therapies into clinical care. London: Royal College of Nursing; 2003.

7. Engebretson J. Alternative and complementary healing: Implications for nursing. J Prof Nurs. 1999; 15:214-23.

8. Melland H, Clayburgh TL. Complementary therapies: Introduction into a nursing curriculum. Nurse Educator. 2000;25:247-50

9. Gordon JS. The Wite House Commisssion on Complementary and Alternative Medicine Policy and the future of Healhcare. Altern Ther Health Med. 2004;10:20-4

10. Helms JE. Complementary and alternative therapies: A new frontier for nursing education? J Nurs Educ. 2006;45:117-24

11. Rankin-Box DF. Paving the way for complementary medicine? (editorial)

Complementary Therapies in Clinical Practice. 2006;12:177-80.

12. Rankin-Box D. The last decade-Complementary therapies in nursing and midwifery. The first decade -Complementary therapies in clinical practice [editorial]. Complementary Therapies Nursing \& Midwifery. 2004;10:205-8

13. VV.AA. Monográfico. Humanitas. Humanidades médicas. Medicinas alternativas y complementarias. 2003;1:31-40 y 79-88

14. Fisher P, Ward A. Complementary medicine in Europe. BMJ. 1994;309:107-11.

15. OMS. El ejercicio de la enfermería. Informe de un comité de expertos de la OMS. Ginebra: OMS; 1995. p. 11-2

16. Fenton M, Morris DL. The integration of holistic nursing practices and complementary and alternative modalities into curricula of schools of nursing. Altern Ther Health Med. 2003;9:62-7.

17. Özge U, Menhtap T. Nursing students' opinions and knowledge about complementary and alternative medicine therapies. Complementary Therapies in Nursing \& Midwifery. 2004;10:239-44.

18. Collière MF. Promover la vida. Madrid: Interamericana-McGraw-Hill; 1993.

19. Breda KL. Teaching complementary healing therapies to nurses. J Nurs Educ. 1998;37:394-97.

20. Colbath JD, Prawlucki PM. Holistic nursing care [preface]. Nurs Clin North Am, 2001;36:XI-XII.

21. López J. Instrumentos complementarios de los cuidados enfermeros: toma de posición. Enfermería Clínica. 2003;13:127-36.22. OIIQ. Les instruments complementaires des soins. I Presse de Position y II Document d'Apuy. Ordre des Infirmières et Infermiers du Québec: 1987; sept.

23. OIIQ. Memoire. Les Practiques Complementaires des Soins. Ordre des Infirmières et Infermiers du Québec: 1993; fevrier
24. Richarson J. Integrating complementary therapies into health care education: a cautious approach. Journal of Clinical Nursing. 2001;10 793-8.

25. Snyder M, Limdquist R. Issues in Complementary Therapies: How We Got to Where We Are. The Online Journal of Issues in Nursing. 2001; 6(2)

26. McCloskey JC, Bulechek GM. Clasificación de Intervenciones de Enfermería. Madrid: Harcourt; 2001.

27. Richardson SF. Complementary health and healing in nursing education. Journal of Holistic Nursing. 2003;21:20-35.

28. Frisch NC. Standards of holistic nursing practice as guidelines for quality undergraduate nursing curricula. J Prof Nurs. 2003;19:382-6.

29. Rankin-Box DF. The Nurses' Hanbook of Complementary Therapies. Edimburgo: Churchill Livingstone; 1995.

\section{Bibliografía complementaria}

Gaydos HL. Aesthetics, art, and holistic nursing. Journal of Holistic Nursing. 2005;23:371.

Gaydos HL. Complementary and alternative therapies in nursing education: Trends and Issues. Online Journal of Issues in Nursing. Artículo publicado el 31 de mayo de 2001. Vol. 6 . Disponible en: http://www.nursingworld.org.

Gordon JS. Manifiesto para una nueva medicina. Barcelona: Paidós; 1997

Isbell B. Clinical training in complementary therapies for 21 st century. Complementary Therapies Nursing \& Midwifery. 2003;9:83-9.

Ort VS, Townsend J. Community-based nursing education and nursing accreditation by the Commission on Collegiate Nursing Education. J Prof Nurs. 2000;16:330-3.

Pepa C, Russell C. Introducing complementary/ alternative strategies in baccalaureate curriculum. Nursing and Health Care Perspectives. 2000;21: 127-29.

Rodriguez M, Rojas MJ, Abreu JB. Enfermería y el presente de las terapias complementarias. ROL de Enfermería. 2002;25:248-52.

Slater V. Holistic Nursing Practice [editorial] Journal of Holistic Nursing. 2005;23:261-2.

Stone J. Regulation of CAM practitioners: reflecting on the last 10 years. Complementary Therapies in Clinical Practice. 2005;11:5-10.

Stuttard P, Walker E. Integrating complementary medicine into the nursing curriculum.

Complementary Therapies Nursing \& Midwifery. 2000;6:87-90.

Trevelyan J, Booth B. Complementary medicine for nurses, midwives and health visitor. Londres: MacMillan Ltd; 1994

Weil A. CAM and continuing education: The future is now. Altern Ther Health Med. 2001;7:32-4.

$\mathrm{Xu}$ Y. Complementary and alternative therapies as philosophy and modalities: Implications for nursing practice, education, and research. Home Health Care Management \& Practice. 2004;16:534-7.

Correspondencia: Julia López Ruiz.

Escola d'Infermeria. UB. Pavelló Central, 3. a planta. Feixa Llarga s/n. 08907 L'Hospitalet de Llobregat. Barcelona. España.

Correo electrónico: julialopez@ub.edu 